\title{
Respondent privacy and estimation efficiency in randomized response surveys for discrete-valued sensitive variables
}

\author{
Mausumi Bose \\ Indian Statistical Institute, Kolkata 700108, India
}

\begin{abstract}
In some socio-economic surveys, data are collected on sensitive or stigmatizing issues such as tax evasion, criminal conviction, drug use, etc. In such surveys, direct questioning of respondents is not of much use and the randomized response technique is used instead. A few researchers have studied the issue of privacy protection or respondent jeopardy for surveys on dichotomous populations, where the objective is to estimate the proportion of persons bearing the sensitive trait. However, not much is yet known about respondent protection when the variable under study takes discrete numerical values and the objective of the survey is to estimate the population mean of this variable. In this article we study this issue. We first propose a randomization device for this situation and give the corresponding estimation procedure. We next propose a measure of privacy and show that given a certain stipulated level of this privacy measure, we can determine the parameter of the randomization device so as to maximize the efficiency of estimation, while guaranteeing the desired level of privacy protection. In particular, our study also covers the case of polychotomous populations and we can estimate the proportions of individuals belonging to the different classes. Consequently, results for dichotomous populations follow as corollaries.
\end{abstract}

Keywords: Jeopardy measure, numerical stigmatizing variable, revealing probability.

\section{Introduction}

The randomized response technique is a useful method for collecting data on variables which are considered sensitive, incriminating or stigmatizing for the respondents. Examples of such situations are common in socio-economic surveys, for instance, we may need to collect data on tax evasion, alcohol addiction, illegal drug use, criminal behaviour or past criminal convictions. In such surveys, direct questions are not useful as the respondents will either refuse to answer embarrassing questions or, even if they do, may give false answers. In a randomized response 
model, the respondents use a randomization device to generate a randomized response and the parameter under study can be estimated from these responses. So, the respondent is not required to disclose his true response and it is expected that this will lead to better participation in the survey on sensitive issues.

Warner (1965) introduced the randomized response technique for estimating the proportion of persons bearing a sensitive attribute in a dichotomous population. In Warner's model, with population categories $A$ and $A^{c}$, a box with two types of cards labeled $A$ and $A^{c}$ (in proportion $p: 1-p)$ is used as the randomization device. A respondent draws a card at random and responds 'yes' or 'no' according as whether or not he belongs to the card type he draws. Since then, several researchers have extensively contributed to this area, e.g., Kuk (1990), Ljungqvist (1993), Mangat (1994), Chua and Tsui (2000), Van den Hout and Van der Heijden (2002), Christofides (2005) and many others. For details on the results available on this technique we refer to the review paper by Chaudhuri and Mukerjee (1987) and books by Chaudhuri and Mukerjee (1988) and Chaudhuri (2011).

Lanke (1976) and Leysieffer and Warner (1976) initiated the study of efficiency versus privacy protection in randomized response surveys where the population is divided into two complementary sensitive groups, $A$ and $A^{c}$, and the objective is to estimate the proportions of persons belonging to these groups. They suggested measures of jeopardy based on the 'revealing probabilities', i.e., the posterior probabilities of a respondent belonging to groups $A$ and $A^{c}$ given his randomized response. Since then, this dichotomous case has been widely studied. Loynes (1976) extended the jeopardy measure of Leysieffer and Warner (1976) to polychotomous populations. Ljungqvist (1993) gave a unified and utilitarian approach to measures of privacy for the dichotomous case. Nayak and Adeshiyan (2009) proposed a measure of jeopardy for surveys from dichotomous populations and developed an approach for comparing the available randomization procedures. These results are all based on samples drawn by simple random sampling with replacement.

All the references given above are for sensitive variables which are categorial or qualitative in nature. However, in randomized response surveys it is quite common to have situations where the study variable $X$ is quantitative, e.g. in studies on the number of criminal convictions of a person, the number of induced abortions, the number of months spent in a correction centre, the amount of undisclosed income, etc. Anderson (1977) studied the case of continuous sensitive variables and considered the amount of information provided by the randomized responses. For 
ensuring more privacy he recommended that the expectation of the conditional variance of $X$ given the randomized response be made as large as possible. However, not much work seems to have been done in studying the respondent privacy aspect for discrete-valued sensitive variables, even though surveys are often undertaken on such variables.

To fill this gap, in this article we focus on studying the issue of privacy protection when the underlying variable under study is quantitative and discrete. We propose the use of a randomization device and give the associated estimation method. Then, we consider two separate cases, one where all values of $X$ are sensitive and another where not all values of $X$ are sensitive. For each of these cases, we propose a measure for protecting the privacy of the respondents. We finally show how one can choose the randomization device parameter in each case, so as to guarantee a certain pre-specified level of respondent protection and then maximize the efficiency of estimating the parameter of interest under this constraint. Our study also covers qualitative sensitive variables, i.e., cases where the population is dichotomous or polychotomous, and allows us to estimate the proportions of individuals belonging to each category.

In Section 2 we give some preliminaries. In Sections 3 and 4 we consider the issues of estimation and privacy protection, respectively. In Section 5 we obtain the randomization device parameter which allows efficient estimation while assuring the required level of respondent protection and illustrate with some numerical examples. In the concluding section we show how our study covers the case of polychotomous variables.

\section{Preliminaries}

Consider a population with $N$ individuals labeled $1, \ldots, N$. Let $X$ denote the sensitive variable of interest. We assume that $X$ takes a finite number of values $x_{1}, \ldots, x_{m}$ and without loss of generality, we may suppose these $m$ values to be known. For $1 \leq i \leq m$, let $\pi_{i}$ be the unknown population proportion of individuals for whom $X$ equals $x_{i}$, i.e.,

$$
\operatorname{Prob}\left(X=x_{i}\right)=\pi_{i}, \quad 1 \leq i \leq m, \quad \text { where } \pi_{\mathrm{i}} \geq 0, \quad \sum_{\mathrm{i}=1}^{\mathrm{m}} \pi_{\mathrm{i}}=1,
$$

The objective of the survey is to estimate the population mean of $X$. For this, we suppose as usual (cf. Warner (1965), Nayak and Adeshiyan (2009) and others), that a sample of $n$ individuals is drawn from the population by simple random sampling with replacement. As for the randomization device, since we are interested in the numerical values of $X$, we propose the use of a device as described below. 
Consider a box containing cards of $(m+1)$ types, the $i$ th type of card being marked 'Report $x_{i}$ as your response', $1 \leq i \leq m$, while the $(m+1)$ th type of card is marked: 'Report your true value of $X$ as your response.' The box has a large number of cards, say $M$, there being $M p$ cards of type $(m+1)$ and $M \frac{1-p}{m}$ cards of each of the types $i, 1 \leq i \leq m, 0<p<1$. A sampled respondent is asked to draw a card at random from the box and then give a truthful response according to the card drawn by him, without disclosing the label on the card to the investigator. Thus the true value of $X$ for the respondent is not known. The $n$ responses so received are the data from this survey.

Let $R$ denote the randomized response variable. Clearly, with this device, the ranges of $R$ and $X$ match. The efficiency in estimation and respondent protection will depend on the choice of the value of $p$, which we call the device parameter. The above device is such that with probability $p$, a respondent will report his true value, while with probability $\frac{1-p}{m}$, he will report any one of the possible values $x_{1}, \ldots, x_{m}$ chosen at random, i.e.,

$$
\begin{aligned}
& \operatorname{Prob}\left(R=x_{i} \mid X=x_{j}\right)=\frac{1-p}{m}, \quad 1 \leq i \neq j \leq m, \\
& \operatorname{Prob}\left(R=x_{j} \mid X=x_{j}\right)=p+\frac{1-p}{m}, \quad 1 \leq j \leq m .
\end{aligned}
$$

\section{Estimation of population mean}

The population mean and variance of $X$ are given by

$$
\mu_{X}=\sum_{i=1}^{m} x_{i} \pi_{i} \text { and } \sigma_{X}^{2}=\sum_{i=1}^{m}\left(x_{i}-\mu_{X}\right)^{2} \pi_{i}
$$

respectively. Our objective is to estimate $\mu_{X}$ from the $n$ randomized responses collected as described in Section 2. Let $w_{i}$ be the sample proportion of randomized responses which equal $x_{i}, \quad 1 \leq i \leq m$. Hence, from (1)-(3),

$$
\mathrm{E}\left(w_{i}\right)=\operatorname{Prob}\left(R=x_{i}\right)=p \pi_{i}+\frac{1-p}{m}=\lambda_{i}, \text { say. }
$$

So, an unbiased estimator of $\pi_{i}$ will be given by $\hat{\pi}_{i}=\frac{1}{p}\left(w_{i}-\frac{1-p}{m}\right)$, leading to an unbiased estimator of $\mu_{X}$ as

$$
\hat{\mu}_{X}=\sum_{i=1}^{m} x_{i} \hat{\pi}_{i}=\frac{1}{p} \sum_{i=1}^{m} x_{i} w_{i}-\frac{1-p}{m p} \sum_{i=1}^{m} x_{i}
$$

Then, on simplification using (4), and writing $\bar{X}=\frac{1}{m} \sum_{i=1}^{m} x_{i}$, the variance of $\hat{\mu}_{X}$ is given by

$$
\operatorname{Var}\left(\hat{\mu}_{X}\right)=\frac{1}{p^{2}} \operatorname{Var}\left(\sum_{i=1}^{m} x_{i} w_{i}\right)=\frac{1}{n p^{2}}\left\{\sum_{i=1}^{m} x_{i}^{2} \lambda_{i}\left(1-\lambda_{i}\right)-\sum \sum_{i \neq j=1}^{m} x_{i} x_{j} \lambda_{i} \lambda_{j}\right\}
$$




$$
\begin{aligned}
& =\frac{1}{n p^{2}}\left\{p \sum_{i=1}^{m} x_{i}^{2} \pi_{i}+\frac{1-p}{m} \sum_{i=1}^{m} x_{i}^{2}-\left(p \mu_{X}+(1-p) \bar{X}\right)^{2}\right\} \\
& =\frac{1}{n p^{2}}\left\{p \sigma_{X}^{2}+(1-p) \frac{1}{m} \sum_{i=1}^{m}\left(x_{i}-\bar{X}\right)^{2}+p(p-1)\left(\mu_{X}-\bar{X}\right)^{2}\right\} .
\end{aligned}
$$

Our aim is to estimate $\mu_{X}$ keeping $\operatorname{Var}\left(\hat{\mu}_{X}\right)$ as small as possible. It is clear from the expression on the right side of (5) that $\operatorname{Var}\left(\hat{\mu}_{X}\right)$ is decreasing in $p$, irrespective of the values of $\pi_{1}, \ldots, \pi_{m}$. So, this variance may be decreased, or equivalently, the efficiency of estimation may be increased by increasing $p$, whatever may be the proportions of the $x_{i}$ values in the population.

\section{Privacy protection}

To study the respondent privacy aspect for dichotomous populations, Leysieffer and Warner (1976) studied the case where both $A$ and $A^{c}$ are sensitive categories while Lanke (1975) also considered the case where only $A$ is sensitive and there is no jeopardy in a 'no' answer to the sensitive question. For polychotomous populations, Loynes (1976) studied two cases, one where all categories are stigmatizing and another where one of the categories is not stigmatizing. In line with these, we too consider the privacy issue for two situations, one where all the $m$ values of $X$ are stigmatizing and another where not all values of $X$ are stigmatizing. Both these situations commonly arise in practice and we require separate privacy protection measures for them.

For a randomly chosen respondent from the population, the 'true' probability that the value of $X$ for this respondent equals $x_{i}$ is given by $\operatorname{Prob}\left(X=x_{i}\right)$. On the other hand, when this respondent gives a randomized response, say $x_{j}$, then the probability that the value of $X$ for this respondent equals $x_{i}$ is now given by the conditional probability $\operatorname{Prob}\left(X=x_{i} \mid R=x_{j}\right)$, or the 'revealing' probability.

\subsection{All values of $X$ are stigmatizing}

Suppose all the values $x_{1}, \ldots, x_{m}$ are stigmatizing. In this case, a respondent would feel comfortable in participating in the survey if the perception of his having a value $X=x_{i}$ is not much altered after knowing his randomized response, for all $1 \leq i \leq m$. This would require that his true and revealing probabilities be sufficiently close. Starting from this basic premise we define

$$
\alpha_{i j}=\left|\operatorname{Prob}\left(X=x_{i} \mid R=x_{j}\right)-\operatorname{Prob}\left(X=x_{i}\right)\right|
$$


and since each respondent would want $\alpha_{i j}$ to be as small as possible for all $1 \leq i, j \leq m$, as a measure of privacy protection we propose the following measure:

$$
\alpha=\max _{1 \leq i, j \leq m} \alpha_{i j}
$$

A randomization device with a privacy protection value $\alpha=\alpha_{0}$ would guarantee that the discrepancies between the true and revealing probabilities will be at most $\alpha_{0}$ for all respondents, irrespective of their true values. Thus a device which results in a lower value of $\alpha$ gives a higher level of privacy protection than one with a higher value of $\alpha$.

Suppose the scientist planning a certain survey would like to keep the privacy protection available to respondents above a certain threshold, i.e., would like to achieve $\alpha \leq \xi$, where $\xi$ is a pre-assigned quantity, $0<\xi<1$. Moreover, this bound on $\alpha$ should hold irrespective of the unknown values of $\pi_{1}, \ldots, \pi_{m}$. The following theorem shows how the device parameter can be chosen to achieve this.

Theorem 1. For $\alpha$ as in (7) and a preassigned $\xi$, where $0<\xi<1, \alpha \leq \xi$ will hold, irrespective of the values of $\pi_{1}, \ldots, \pi_{m}$, if and only if $p \leq p_{0}$, where

$$
p_{0}=\frac{1}{1+\frac{m}{\xi}\left(\frac{1-\xi}{2}\right)^{2}} .
$$

Proof. From (1)-(3), using Bayes' Theorem it follows that for $1 \leq i, j, \leq m$,

$$
\operatorname{Prob}\left(X=x_{i} \mid R=x_{j}\right)=\frac{\left(p \delta_{i j}+\frac{1-p}{m}\right) \pi_{i}}{\sum_{u=1}^{m}\left(p \delta_{j u}+\frac{1-p}{m}\right) \pi_{u}}=\frac{\left(p \delta_{i j}+\frac{1-p}{m}\right) \pi_{i}}{p \pi_{j}+\frac{1-p}{m}},
$$

where $\delta_{i j}$ is Kronecker Delta. Hence from (6) it follows that $\alpha_{i j}=\frac{p \pi_{i}\left|\pi_{j}-\delta_{i j}\right|}{p \pi_{j}+\frac{1-p}{m}}$ and for any $i \neq j$,

$$
\alpha_{i j}=\frac{p \pi_{i} \pi_{j}}{p \pi_{j}+\frac{1-p}{m}} \leq \frac{p\left(1-\pi_{j}\right) \pi_{j}}{p \pi_{j}+\frac{1-p}{m}}=\alpha_{j j}
$$

as $\pi_{i}+\pi_{j} \leq 1$ for all $i, j$. Thus $\alpha=\max _{1 \leq j \leq m} \alpha_{j j}=\max _{1 \leq j \leq m} \frac{\pi_{j}\left(1-\pi_{j}\right)}{\pi_{j}+\frac{1-p}{m p}}$. Hence, $\alpha \leq \xi$ if and only if

$$
\pi_{j}\left(1-\pi_{j}\right)-\xi \pi_{j} \leq \frac{\xi(1-p)}{m p} \text { for all } 1 \leq j \leq m .
$$

First suppose $p \leq p_{0}$. Then for $1 \leq j \leq m$,

$$
\begin{aligned}
\pi_{j}\left(1-\pi_{j}\right)-\xi \pi_{j} & =\left(\frac{1-\xi}{2}\right)^{2}-\left(\frac{1-\xi}{2}-\pi_{j}\right)^{2} \\
& \leq\left(\frac{1-\xi}{2}\right)^{2}=\frac{\xi\left(1-p_{0}\right)}{m p_{0}}, \text { using } \\
& \leq \frac{\xi(1-p)}{m p} .
\end{aligned}
$$


Thus the inequalities in (11) hold, or equivalently $\alpha \leq \xi$, irrespective of the values of $\pi_{1}, \ldots, \pi_{m}$.

To prove the converse, suppose $\alpha \leq \xi$, or equivalently, the inequalities in (11) hold, irrespective of the values of $\pi_{1}, \ldots, \pi_{m}$. Then, for $\pi_{1}=\frac{1-\xi}{2}, \pi_{2}=\frac{1+\xi}{2}, \pi_{3}=\ldots=\pi_{m}=0$, in particular, these inequalities will also hold. So, for this choice of $\pi_{j}$ values in (11) with $j=1$, we have

$$
\begin{aligned}
\left(\frac{1-\xi}{2}\right)\left(\frac{1+\xi}{2}\right)-\xi\left(\frac{1-\xi}{2}\right) & \leq \frac{\xi(1-p)}{m p} \\
\text { i.e., }\left(\frac{1-\xi}{2}\right)^{2} & \leq \frac{\xi(1-p)}{m p}
\end{aligned}
$$

So from (8), $p \leq p_{0}$. Hence theorem.

Remark 1. It is clear from (8) that in order to maintain the same level of protection, the value of $p_{0}$ monotonically decreases with the number of possible values of $X$. Again, for a given number of possible values of $X, p_{0}$ monotonically increases with $\xi$. We may reiterate that these values of $p$ do not depend on how the values of $X$ are distributed in the population.

\subsection{Not all values of $X$ are stigmatizing}

In many surveys it may so happen that not all values of $X$ are sensitive or stigmatizing. For instance, in a survey for estimating the average number of criminal convictions of persons in a certain population, the value $X=0$ is not stigmatizing but any value of $X \geq 1$ could well be stigmatizing. Similarly, for a survey for estimating the average of the number (X) of induced abortions, the values $X=0$ or $X=1$ might not be considered as stigmatizing values while other larger values might be considered stigmatizing by the respondents.

To study the respondents' privacy protection for such surveys, we present here the simpler case where only one of the values of $X$, say $x_{1}$, is not stigmatizing, while values $x_{2}, \ldots, x_{m}$ are considered stigmatizing. We develop the protection measure for this case in detail. Later we remark that the results obtained for this case may be easily extended to the case where $X$ has more than one non-stigmatizing values.

As before, the data collection and estimation proceeds as in Sections 2 and 3. To study the respondent protection we note that since the value $x_{1}$ is non-stigmatizing, respondents will feel comfortable with a randomization device for which the 'revealing' probability of their having a true value $x_{1}$ will be large. So, we propose the following measure of privacy:

$$
\beta=\min _{1 \leq j \leq m} P\left(X=x_{1} \mid R=x_{j}\right)=\min _{1 \leq j \leq m} \frac{\left(p \delta_{1 j}+\frac{1-p}{m}\right) \pi_{1}}{p \pi_{j}+\frac{1-p}{m}},
$$


on simplification using (9). A device with a privacy protection value $\beta$ will guarantee that all respondents are perceived to have $X=x_{1}$ with probability at least $\beta$. So, a device leading to a larger value of $\beta$ will ensure greater privacy to respondents than one with a smaller $\beta$.

Let $\xi, 0<\xi<1$, denote a preassigned level of respondents' privacy. Then in order to achieve this level of protection we require that $\beta \geq \xi$, irrespective of the values of $\pi_{1}, \ldots, \pi_{m}$. Thus we should have

$$
\left(p \delta_{1 j}+\frac{1-p}{m}\right) \pi_{1} \geq \xi\left(p \pi_{j}+\frac{1-p}{m}\right), \quad 1 \leq j \leq m,
$$

or equivalently, the following inequalities should hold:

$$
\begin{aligned}
& {\left[p(1-\xi)+\frac{1-p}{m}\right] \pi_{1} \geq \frac{\xi(1-p)}{m}} \\
& \text { and } \frac{1-p}{m} \pi_{1}-\xi p \pi_{j} \geq \frac{\xi(1-p)}{m}, \quad 2 \leq j \leq m .
\end{aligned}
$$

Clearly, no $p$ can satisfy (13) irrespective of $\pi_{1}, \ldots, \pi_{m}$ for any given $\xi$ since (13) fails as $\pi_{1} \rightarrow 0$. So we assume that $\pi_{1}>0$ and we also assume some prior knowledge about a lower bound on $\pi_{1}$. This assumption is quite realistic because in most populations there will be an appreciable number of persons with a non-stigmatizing variable value and hence, a lower bound to the proportion of such stigma-free persons in the population will be available.

Thus, suppose we have prior knowledge that $\pi_{1} \geq c$. We work with $\xi<c$. This is again realistic because if the only knowledge about $\pi_{1}$ is that $\pi_{1} \geq c$, it is impractical to demand that $P\left(X=x_{1} \mid R=x_{j}\right) \geq \xi(\geq c)$ for all $j$. Now, the following theorem gives the value of the device parameter $p$ which will guarantee the desired level of respondent protection $\xi$.

Theorem 2. Let $\beta$ be as in (12) and $\pi_{1} \geq c$ for some known $c$. Then given a preassigned $\xi$, where $0<\xi<c, \beta \geq \xi$ will hold, irrespective of the values of $\pi_{1}, \ldots, \pi_{m}$, if and only if $p \leq p_{0}$, where

$$
p_{0}=\frac{\frac{c-\xi}{m}}{\frac{c-\xi}{m}+\xi(1-c)} .
$$

Proof. Since $\pi_{1} \geq c$, it is clear that $\pi_{j} \leq 1-c$ for $2 \leq j \leq m$ and we have

$$
\begin{aligned}
& {\left[p(1-\xi)+\frac{1-p}{m}\right] \pi_{1} \geq\left[p(1-\xi)+\frac{1-p}{m}\right] c} \\
& \text { and } \frac{1-p}{m} \pi_{1}-\xi p \pi_{j} \geq \frac{1-p}{m} c-\xi p(1-c), \quad 2 \leq j \leq m .
\end{aligned}
$$

As a result, (13) and (14) will hold, irrespective of the true values of $\pi_{1}(\geq c), \pi_{2}, \ldots, \pi_{m}$ iff

$$
\text { and } \begin{aligned}
{\left[p(1-\xi)+\frac{1-p}{m}\right] c } & \geq \xi \frac{1-p}{m} \\
\frac{1-p}{m} c-\xi p(1-c) & \geq \xi \frac{1-p}{m}
\end{aligned}
$$


hold. Now, (16) reduces to

$$
\left(p+\frac{1-p}{m}\right) c \geq \xi\left(c p+\frac{1-p}{m}\right)
$$

which will always hold for every $p$ since $\xi\left(c p+\frac{1-p}{m}\right) \leq \xi\left(p+\frac{1-p}{m}\right)<c\left(p+\frac{1-p}{m}\right)$ as $\xi<c$ and $p+\frac{1-p}{m}>0$. So, it is enough to only consider $(17)$. Note that

$$
\begin{aligned}
(17) \Leftrightarrow \frac{c-c p}{m}-\xi p(1-c) & \geq \frac{\xi-\xi p}{m} \\
\Leftrightarrow p & \leq=\frac{\frac{c-\xi}{m}}{\frac{c-\xi}{m}+\xi(1-c)}=p_{0},
\end{aligned}
$$

thus proving the theorem.

Remark. The above discussion can be extended to include the more general case where $X$ has $t$ non-stigmatizing values $x_{1}, \ldots, x_{t}$, say, while its remaining $m-t$ values are stigmatizing, $1<t<m$. In that case too, it can be shown that $p_{0}$ takes the form as in Theorem 2 , but now with

$$
\beta=\min _{1 \leq j \leq m} P\left(X=x_{1} \text { or } x_{2} \text { or } \ldots x_{t} \mid R=x_{j}\right) \text { and } \pi_{1}+\ldots+\pi_{t} \geq c \text { with } \xi<c .
$$

\section{Privacy protection together with efficiency in estimation}

We now consider the issue of efficiency in estimation together with privacy protection in randomized response surveys. It was seen from (5) that, irrespective of the values of $\pi_{1}, \ldots, \pi_{m}$, the efficiency of estimation may be increased by increasing $p$. On the other hand, for a given $\xi$ and irrespective of the values of $\pi_{1}, \ldots, \pi_{m}$, Theorems 1 and 2 show that a protection of $\alpha \leq \xi$ or $\beta \geq \xi$ may be guaranteed iff $p \leq p_{0}$, where $p_{0}$ is as in (8) or (15), respectively. So, the best choice of $p$ with regard to maximizing the efficiency of estimation of $\mu_{X}$, subject to the stipulated level of privacy protection $\xi$, is $p=p_{0}$. The following examples illustrate this.

Example 5.1 Let $X$ take four values which are all sensitive. Suppose $\xi=0.1$ Then by Theorem $1, p_{0}=0.1099$. So, if we use a randomization device with $p=0.1099$ then the efficiency of estimation can be maximized while guaranteeing that the maximum discrepancy between the true probability and the revealing probability of all respondents will be at most 0.1 .

The following table gives the $p_{0}$ values in (8) for some choices of $\xi$ and $m$. 


\begin{tabular}{|c|c|c|c|c|c|c|c|c|c|}
\hline$m$ & $\xi$ & $p_{0}$ & $m$ & $\xi$ & $p_{0}$ & & $m$ & $\xi$ & $p_{0}$ \\
\hline 3 & 0.1 & 0.1413 & 4 & 0.1 & 0.1099 & 5 & 0.1 & 0.0899 \\
3 & 0.2 & 0.2941 & 4 & 0.2 & 0.2381 & 5 & 0.2 & 0.2000 \\
3 & 0.3 & 0.4494 & 4 & 0.3 & 0.3797 & 5 & 0.3 & 0.3288 \\
3 & 0.4 & 0.5970 & 4 & 0.4 & 0.5263 & 5 & 0.4 & 0.4706 \\
\hline
\end{tabular}

Example 5.2 Let $X$ take one nonsensitive value and two sensitive values. Suppose it can be assumed that at least $15 \%$ of the individuals in the population possess the nonsensitive value and suppose it is stipulated that $\xi=0.10$. Then by Theorem $2, p_{0}=0.1639$. So, if we use a device with $p=0.1639$ then estimation efficiency will be maximum while guaranteeing that all respondents will have at least a $10 \%$ probability of being revealed as belonging to the nonstigmatizing class.

\section{Estimation of population proportions}

As mentioned in Section 1, several researchers have estimated the proportions of individuals belonging to the two categories in dichotomous populations, while Loynes (1976) extended this to estimating the different proportions in a polychotomous population. In our case where $X$ takes $m$ numerical values, we may also readily estimate the population proportions $\pi_{1}, \ldots, \pi_{m}$ from the responses collected as in Section 2 and again use the measures of privacy as given in (7) and (12) to achieve the stipulated level of privacy protection.

As seen in Section 3, an unbiased estimate of $\pi_{i}$ is

$$
\hat{\pi}_{i}=\frac{1}{p}\left(w_{i}-\frac{1-p}{m}\right), \quad 1 \leq i \leq m
$$

Suppose, in the spirit of $A$-optimality commonly used in optimal design theory, we would like to minimize the average variance of these estimates. For this, we can show that

$$
\sum_{i=1}^{m} \operatorname{Var}\left(\hat{\pi}_{i}\right)=\frac{1}{n p^{2}} \sum_{i=1}^{m} \lambda_{i}\left(1-\lambda_{i}\right)=\frac{1}{n}\left\{\frac{1}{p^{2}}-\sum_{i=1}^{m} \pi_{i}^{2}+\frac{1}{m}\left(\frac{1}{p^{2}}-1\right)\right\},
$$

on simplification, using (4). Clearly, (18) is decreasing in $p$, irrespective of the true values of $\pi_{1}, \ldots, \pi_{m}$. So as in the case of estimating the mean, here too, given some $\xi$, subject to the constraint on protection of privacy, the best choice for $p$ for minimizing the average variance of the estimates of the proportions, is $p=p_{0}$, with $p_{0}$ being given by (8) or (15), as the case may be. The popular case of dichotomous populations follow by taking $m=2$ in the above. 


\section{References}

Anderson, H. (1977) Efficiency versus protection in a general randomized response model. Scand. J. Statist. 4, 11-19.

Chaudhuri, A. (2011) Randomized response and indirect questioning techniques in surveys. CRC Press, Boca Raton, FL.

Chaudhuri, A. and Mukerjee, R. (1987) Randomized response techniques: a review. Statist. Neerlandica 41 1, 27-44.

Chaudhuri, A. and Mukerjee, R. (1988) Randomized responses: Theory and Techniques. Marcel Dekker, New York, NY.

Christofides, T.C., (2005) Randomized response in stratified sampling. J. Statist. Plann. Inference $128,303-310$.

Chua, T.C. and Tsui, A.K. (2000) Procuring honest responses indirectly. J. Statist. Plann. Inference 90, 107-116.

Kuk, A.Y.C. (1990) Asking sensitive questions indirectly. Biometrika 77, 436-438.

Lanke, J. (1975) On the choice of the unrelated question in Simmons' version of randomized response. J. Amer. Statist. Assoc. 70, 80-83.

Lanke, J. (1976) On the degree of protection in randomized interviews. Int. Stat. Rev. 44, 197-203.

Leysieffer, R.W. and Warner, S.L. (1976) Respondent jeopardy and optimal designs in randomized response models. J. Amer. Statist. Assoc. 71, 649-656.

Ljungqvist, L. (1993) A unified approach to measures of privacy protection in randomized response models: a utilitarian perspective. J. Amer. Statist. Assoc. 88, 97-103.

Loynes, R.M. (1976) Asymptotically Optimal Randomized Response Procedures. J. Amer. Statist. Assoc. 71, 924-928.

Mangat, N.S. (1994) An improved randomized response strategy. J. Roy. Statist. Soc. 56, 93-95.

Nayak, T. K. and Adeshiyan, S. A. (2009) A unified framework for analysis and comparison of randomized response surveys of binary characteristics. J. Statist. Plann. Inf. 139, $2757-2766$.

Van den Hout, A. and Van der Heijden, P.G.M. (2002) Randomized response, statistical disclosure control and misclassification: a review. Internat. Statist. Rev.70, 269-288. 
Warner, S.L. (1965). Randomized response: a survey technique for eliminating evasive answer bias. J. Amer. Statist. Assoc. 60, 63-69. 